\title{
Looking into the genetic bases of OCD dimensions: a pilot genome-wide association study
}

María Alemany-Navarro (10 ${ }^{1,2,3}$, Raquel Cruz ${ }^{4}$, Eva Real ${ }^{1,2,5}$, Cinto Segalàs ${ }^{1,2,5}$, Sara Bertolín (1) ${ }^{2}$, Raquel Rabionet ${ }^{6,7}$, Ángel Carracedo ${ }^{4,8}$, Jose M. Menchón (1) ${ }^{1,2,3,5}$ and Pino Alonso $2,3,5$

\begin{abstract}
The multidimensional nature of obsessive-compulsive disorder (OCD) has been consistently reported. Clinical and biological characteristics have been associated with OCD dimensions in different ways. Studies suggest the existence of specific genetic bases for the different OCD dimensions. In this study, we analyze the genomic markers, genes, gene ontology and biological pathways associated with the presence of aggressive/checking, symmetry/order, contamination/cleaning, hoarding, and sexual/religious symptoms, as assessed via the Dimensional Yale-Brown Obsessive Compulsive Scale (DY-BOCS) in 399 probands. Logistic regression analyses were performed at the singlenucleotide polymorphism (SNP) level. Gene-based and enrichment analyses were carried out for common (SNPs) and rare variants. No SNP was associated with any dimension at a genome-wide level $\left(p<5 \times 10^{-8}\right)$. Gene-based analyses showed one gene to be associated with hoarding (SETD3, $p=1.89 \times 10^{-08}$ ); a gene highly expressed in the brain and which plays a role in apoptotic processes and transcriptomic changes, and another gene associated with aggressive symptoms ( $C P E ; p=4.42 \times 10^{-6}$ ), which is involved in neurotrophic functions and the synthesis of peptide hormones and neurotransmitters. Different pathways or biological processes were represented by genes associated with aggressive (zinc ion response and lipid metabolism), order (lipid metabolism), sexual/religious (G protein-mediated processes) and hoarding (metabolic processes and anion transport) symptoms after FDR correction; while no pathway was associated with contamination. Specific genomic bases were found for each dimension assessed, especially in the enrichment analyses. Further research with larger samples and different techniques, such as next-generation sequencing, are needed to better understand the differential genetics of OCD dimensions.
\end{abstract}

\section{Background}

Obsessive-compulsive disorder (OCD) is a neuropsychiatric condition that has an estimated prevalence of $2-3 \%{ }^{1}$. Despite the unitary nosological status of OCD (DSM-5), considerable heterogeneity of OCD symptoms exists. Several studies have looked into different symptom dimensions present in OCD; some have reported exploratory or confirmatory factor analysis based on Yale-Brown Obsessive Compulsive Scale Checklist (Y-BOCS-CL) items.

Correspondence: María Alemany-Navarro (mariaalemany92@gmail.com) ${ }^{1}$ Institut d'Investigació Biomèdica de Bellvitge (IDIBELL), L'Hospitalet de Llobregat, Barcelona, Spain

${ }^{2}$ OCD Clinical and Research Unit, Psychiatry Department, Hospital Universitari de Bellvitge, Barcelona, Spain

Full list of author information is available at the end of the article
Most of those studies have reported four or five main (second-order) factors, which in part depend on the methodology employed ${ }^{2,3}$, thereby suggesting a multidimensional model for OCD. This multidimensional nature of OCD has been confirmed by meta-analyses and systematic reviews ${ }^{4,5}$. Along these lines, specific instruments such as the Dimensional Yale-Brown Obsessive Compulsive Scale (DY-BOCS ${ }^{6}$ ) have been developed to assess OCD severity in different symptom dimensions.

A range of clinical characteristics has been associated with OCD symptom dimensions in different ways. In this vein, it has been proposed that the hoarding and symmetry dimensions are characteristic of an early-age OCD group $^{7,8}$. In terms of comorbidities, a factor comprising aggressive, sexual and religious symptoms has been 
associated with comorbid major depressive disorder and bipolar disorder (MDD/BD); while patients with symmetry/order symptoms show greater comorbidity with eating and addictive disorders as well as attention-deficit/ hyperactivity disorder (ADHD) ${ }^{9}$. Meanwhile, the contamination/cleaning dimension has been reported as the dimension that is least frequently associated with any other Axis I disorder ${ }^{10}$. In addition, the hoarding and symmetry/order dimensions have been associated with a poorer response to pharmacological treatment ${ }^{8,10}$.

Differential endophenotypic profiles have also been reported in relation to symptom dimensions in neuroimaging studies. For instance, OCD probands with symmetry/order symptoms have been reported to present a reduced volume of the right precentral gyrus ${ }^{11}$ and the hippocampus, which is also associated with aggressive/ checking symptoms when compared to healthy controls ${ }^{12}$. In addition, the aggressive/checking and contamination/ cleaning dimensions have been negatively correlated to right cerebellum and right insula volumes, respectively ${ }^{13}$. In terms of functionality, patients with aggressive/checking and sexual/religious symptoms have been found to present greater amygdala activation when confronted by fear-inducing stimuli ${ }^{14}$. Also, differences in connectivity have been observed between the aggressive/checking, sexual/religious and hoarding dimensions ${ }^{15}$. Although we do not know to what extent these observed neurological differences between the OCD dimensions have a genetic basis, some of the structural and functional brain characteristics identified have been directly related to certain genetic variants in OCD patients as well as in those with other psychiatric disorders ${ }^{16-20}$.

In fact, specific genetic bases have been identified for the different OCD symptom dimensions. For instance, severity of the contamination/cleaning dimension has been associated with both the Met allele of the Vall66Met locus within the brain-derived neurotrophic factor gene $(B D N F)^{21}$ and the c.256G allele of the 5hydroxytryptamine receptor $3 \mathrm{E}$ (HTR3E) variant rs7627615 $5^{22}$. The presence of this dimension has also been associated with the variants rs4657411 within the LIM homeobox transcription factor 1 alpha gene (LMX1A), and rs2075507 of the catechol-O-methyltranspherase gene (COMT) in women ${ }^{23,24}$. In addition, a protective role against these dimensions has been attributed to the ACCCG haplotype of the estrogen receptor 1 gene $(E S R 1)^{25}$. The symmetry/order dimension has been related to the $S$ allele and the SS genotype of the serotonin transporter polymorphic region (SERTPR) ${ }^{26}$; the presence of the $2 \mathrm{R}$ allele within the dopamine receptor $\mathrm{D} 4$ gene (DRD4) 48-bp variable number of tandem repeats polymorphism $(\mathrm{VNTR})^{27}$; and the A allele in the COMT variant rs2075507 in men ${ }^{24}$. This dimension in combination with aggressive/checking behavior has been associated with specific variants in a promoter region of the glutamate ionotropic receptor NMDA-type subunit 2B gene (GRIN2B) $\left(\right.$ rs1019385) ${ }^{28}$ and the SLIT and NTRK-like family member 1 gene (SLITRK1) (rs9593835); the latter, specifically in $\operatorname{men}^{23}$. The SERTPR has also been associated with higher scores in a religious/somatic dimension ( $1 / \mathrm{s}$ and $\mathrm{l} / \mathrm{l}$ genotypes) ${ }^{29}$ and in counting and repeating rituals in OCD patients with a comorbid tic disorder ( $1 / 1$ genotype $)^{30}$. Women who exhibit hoarding symptoms have been reported to present a higher frequency of the Met/Met genotype of the COMT variant rs4680 than those who do not. Hoarding has also been associated with a variant (rs1017412) within the neurotrophic receptor tyrosine kinase 3 gene $(N T R K)^{31}$ and with both the short variant of the serotonin-transporterlinked polymorphic region (5HTTLPR), and its long variant together with the $\mathrm{G}$ allele at rs 25531 in males ${ }^{32}$. A neutralization dimension, as assessed by the ObsessiveCompulsive Inventory-Revised (OCI-R) has been associated with a variant of $L M X 1 A$ (rs4657411) ${ }^{23}$. Severity scores in a dimension comprising somatic and sensory phenomena symptoms have shown a trend towards an association with the Val58Met genotype of the COMT gene in interaction with sex, with women presenting lower scores ${ }^{33}$.

Although a large number of studies have focused on elucidating the genetic basis of OCD, inconsistent results have been reported in most respects ${ }^{34}$. A possible explanation for this is that the studies do not usually consider different symptom profiles among OCD patients. It has consistently been argued that it is necessary to account for OCD heterogeneity in genetic and neurobiological studies $^{35,36}$. Therefore, in this study, we analyze the variants, genes and functional pathways that might be differentially involved in the OCD dimensions measured by the DYBOCS $^{6}$ through an exploratory genomic method. We hypothesize that different genomic bases will be found for the different OCD dimensions.

\section{Methods \\ Subjects}

Three hundred and ninety-nine Caucasian Spanish patients $(n=399$; 210 women; mean age $=35 \pm 11)$ with an OCD diagnosis were recruited from the OCD clinic at Bellvitge Hospital (Barcelona, Spain). Diagnoses were made by three psychiatrists with extensive clinical experience in OCD, following the DSM-IV criteria for OCD diagnosis ${ }^{37}$ using the Structured Clinical Interview for DSM-IV Axis I Disorders-Clinician Version $(\mathrm{SCIDCV})^{38}$. All the patients had the disorder for at least one year. Those patients presenting psychoactive substance abuse/dependence (current or in the past six months), psychotic disorders, intellectual disability, severe organic or neurological pathology (except tic disorders), 
or autism spectrum disorders were excluded from the study. Other affective and anxiety disorders were not criteria for exclusion in cases where OCD was the main diagnosis.

Participants were required to give written consent after being fully informed about the study. The study was approved by the Ethical Committees of Bellvitge Hospital and was performed in accordance with the Helsinki Declaration of the World Medical Association.

\section{Clinical assessment}

Medical data and both sociodemographic and clinical characteristics were collected via a structured interview during each patient's first appointment at the clinic.

Age at onset was defined as the moment when obsessive symptoms reached a clinically significant level. Family psychiatric history was considered dichotomously, but specific information regarding family history of OCD, Tourette syndrome and depression was also collected. Only family members who had received a formal diagnosis were considered to be affected.

Baseline severity of obsessive and compulsive symptoms was also assessed through the clinician-administered version of the Y-BOCS ${ }^{39}$ during the patient's first visit to the clinic. A global measure, as well as independent measures for both obsessions and compulsions, were recorded.

\section{$O C D$ Dimension: presence and severity}

Dimension-specific presence and severity were evaluated using the DY-BOCS ${ }^{6}$, which is composed of a selfreport part and a clinician-rated instrument. It assesses OCD severity in six different symptom dimensions that gather together thematically similar symptoms. The six are: aggressive obsessions and checking compulsions (aggressive/checking); symmetry obsessions and order compulsions (symmetry/order); contamination obsessions and cleaning/washing compulsions (contamination/ cleaning); hoarding obsessions and compulsions (hoarding); sexual or religious obsessions accompanied by different rituals (sexual/religious); and a miscellaneous dimension including other obsessive thoughts and compulsive behavior (miscellaneous). We did not consider this last dimension in our analyses given its lack of specificity.

\section{Genotyping data and quality control}

Our sample consisted of 399 OCD patients genotyped using the Infinium PsychArray-24 BeadChip from Illumina. This array was developed in collaboration with the Psychiatric Genomics Consortium (PGC) and includes 50,000 variants associated with common psychiatric disorders. Variant calling was performed using three different algorithms: GenCall, which is Illumina's default calling algorithm, and Birdseed, both for common variants; and zCall, aimed at rare variant calling. A unique set of genotypes was derived from a consensus merge of the GenCall and Birdseed common variants, also including rare variants called by $\mathrm{zCall}$ that passed quality control (QC) from the consensus merge of GenCall and Birdseed.

QC filtering of genotype data was performed using PLINK $^{40}$. Only non-monomorphic autosomal biallelic variants in Hardy Weinberg equilibrium $(p<0.0001)$ with a call rate of above $98 \%$ were included.

Samples that had a call rate lower than $98 \%$ were removed. Identity by descent was calculated using independent SNPs, and omitting those samples with a pi-hat greater than $0.2^{41}$. Population stratification was tested by principal component analysis, removing those samples that deviated by more than 5 standard deviations (SDs) from the mean in the first two components.

\section{Statistical analysis}

Association analyses at the SNP level were performed using the GenABEL library for R software ${ }^{42}$. Regression analyses were carried out under a log-additive model (in which the genotypes were coded as 0,1 or 2 depending on the number of minor alleles). These operations were performed for autosomal SNPs (markers showing a minor allele frequency (MAF) $>0.05$ in autosomes). Given the non-normality of the DY-BOCS scores and the impossibility of normalizing them, these variables were dichotomized to analyze the presence/absence of the different DY-BOCS dimensions. A logistic regression analysis was performed for each dimension, with the dependent variable being the dimension we were testing. Age, sex, and the four other dimensions were included as covariates in all the models. Linkage-disequilibrium (LD) plots were designed using the LocusZoom software, based on 1000 genome CEU population data (hg19/1000 Genomes Mar 2012 EUR $)^{43}$. For SNP annotation, we used the Infinium PsychArray Gene Annotation File provided by Illumina (https://support.illumina.com/downloads/ infinium-psycharray-product-support-files.html).

Power calculations were performed with Genetic Association Study Power Calculator software (http://csg.sph. umich.edu/abecasis/cats/gas_power_calculator/reference. html) to determine the power of our study to detect associations considering our sample size.

Gene-based association analyses were performed via the Sequence Kernel Association Test (SKAT) ${ }^{44}$ using the SKATMeta library ${ }^{45}$ for $\mathrm{R}$ software. This type of genebased analysis has the advantage of including rare variants, which were not considered in SNP-based analyses given the lack of statistical power for detecting associations with these variants at a single-variant level. SKAT combines the effects of common and rare variants in gene-sets, increasing the power to detect small effects. 
Only results from genes with at least two genotyped markers were considered. A false discovery rate (FDR) correction was used as a significance criterion.

Finally, enrichment analyses were performed for genes with at least two genotyped markers that had a SKAT $p$ value lower than 0.01 using web-accessible DAVID (Database for Annotation, Visualization, and Integrated Discovery) Bioinformatic Resources v6. $8^{46,47}$. This software analyzes input genes in the context of a genomic background (in this study, we selected the entire human genome as background) to cluster genes enriched in biological pathways and gene ontologies. We ordered the reported results by the FDR statistic to prioritize for further interpretation.

\section{Results}

\section{Subjects and genotyping quality control}

Three hundred and seventy-six $(n=376)$ samples passed quality control procedures. Table 1 summarizes the sociodemographic and clinical data including DY-BOCS scores gathered for the final OCD sample.

\section{SNP-level association analyses}

Our total dataset consisted of 338,357 autosomal markers, of which 258,937 were SNPs (MAF $\geq 0.05$ ).

No SNP exceeded the statistical threshold for genomewide significance $\left(p<5 \times 10^{-8}\right)$ in any dimension (Fig. 1). The results at a $p$ value $\leq 10^{-4}$ for the five dimensions can be seen in Table 2. Suggestive associations $\left(p<10^{-5}\right)$ were found with the aggressive, contamination, order, and hoarding dimensions (Fig. 1a-d).

In the order dimension, eight variants presented suggestive associations $\left(p<10^{-5}\right)$. Six of these variants were within chromosome 12 and, given the proximity between them (5 of them were variants of the IPO8 gene), we performed LD analysis on this region. We found two clusters of SNPs: (1) rs7316477 $\left(1.50 \times 10^{-6}\right)$, rs14139 $\left(1.50 \times 10^{-6}\right), \quad \operatorname{rs} 6487927\left(1.58 \times 10^{-6}\right)$ and $\operatorname{rs} 10771752$ $\left(1.83 \times 10^{-6}\right)$, all exonic variants located in the IPO8 (Importin 8) gene (Fig. 2a); and (2) rs6487928 (2.99 $\times$ $\left.10^{-6}\right)$ and rs12146709 $\left(3.70 \times 10^{-6}\right)$, exonic variants located in IPO8 and CAPRIN2 (Caprin Family Member 2), respectively (Fig. 2b). All these markers should be considered as forming a single association peak, since the level of association was not maintained for any of them when adjusting the model for each of the other markers.

\section{Gene-based association analyses}

Gene-based analysis were performed for 22,017 (aggressive), 21,179 (order), 20,466 (contamination) and 22,472 (hoarding and sexual/religious) genes. Of these, 17,001 (aggressive), 16,467 (order), 15,790 (contamination), and 17,378 (hoarding and sexual/religious) had at least two genotyped markers and were considered in our further analyses.
Table 1 Sociodemographic and clinical characteristics of the sample of 376 OCD patients.

\begin{tabular}{|c|c|}
\hline Age, years & $35.2 \pm 10.7(18-71)$ \\
\hline Male/Female & 186/190 (49.5/ 50.5) \\
\hline Age at onset of OCD & $19.9 \pm 8.9(4-46)^{\mathrm{a}}$ \\
\hline \multicolumn{2}{|l|}{ Y-BOCS score } \\
\hline Global & $25.8 \pm 5.5(9-40)$ \\
\hline Obsessions & $12.6 \pm 3.6(0-20)$ \\
\hline Compulsions & $12.3 \pm 4.0(0-20)$ \\
\hline Baseline HDRS score & $12.2 \pm 6.0(0-29)$ \\
\hline \multicolumn{2}{|l|}{ Current comorbidity } \\
\hline No comorbidity & $212(56.4)$ \\
\hline Mood disorder & $71(18.9)$ \\
\hline Tics & $52(13.8)$ \\
\hline Eating disorders & $19(05.1)$ \\
\hline \multicolumn{2}{|c|}{ Presence of dimensions in worst-ever period } \\
\hline Aggresive/checking & $278(73.9)$ \\
\hline Symmetry/ordering & $162(43.1)$ \\
\hline Contamination/cleaning & $172(45.7)$ \\
\hline Hoarding & $91(24.2)$ \\
\hline Sexual/religious & $95(25.3)$ \\
\hline \multicolumn{2}{|l|}{ Family psychiatric history } \\
\hline No psychiatric diagnosis & $138(36.7)$ \\
\hline OCD & $81(21.5)$ \\
\hline Mood disorder & $114(30.3)$ \\
\hline Tics/ Tourette sydrome & $35(9.3)$ \\
\hline
\end{tabular}

Data are mean \pm SD (range) or percentage (\%).

$O C D$ obsessive compulsive disorder, $Y$-BOCS Yale-Brown Obsessive Compulsive Scale, HDRS Hamilton Depression Rating Scale.

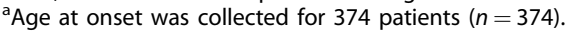

Our results for genes with at least a suggestive association $\left(p<10^{-4}\right)$ in the five dimensions can be seen in Table 3. One gene reached genome-wide significant association with hoarding (SET Domain Containing 3, Actin Histidine Methyltransferase, SETD3; $p=1.89 \times$ $10^{-08}$ ). This gene codes for a protein involved, among other functions, in actin binding and modification, histone methylation, chromatin organization, and regulation of transcription ${ }^{48}$. The second most significant gene was $C P E$ (Carboxypeptidase E), which reached genomewide significant association with the aggressive dimension $\left(p=4.42 \times 10^{-6}\right)$. This gene codes for a membrane protein involved in the synthesis of peptide hormones and neurotransmitters as well as different neurotrophic functions ${ }^{49}$. 

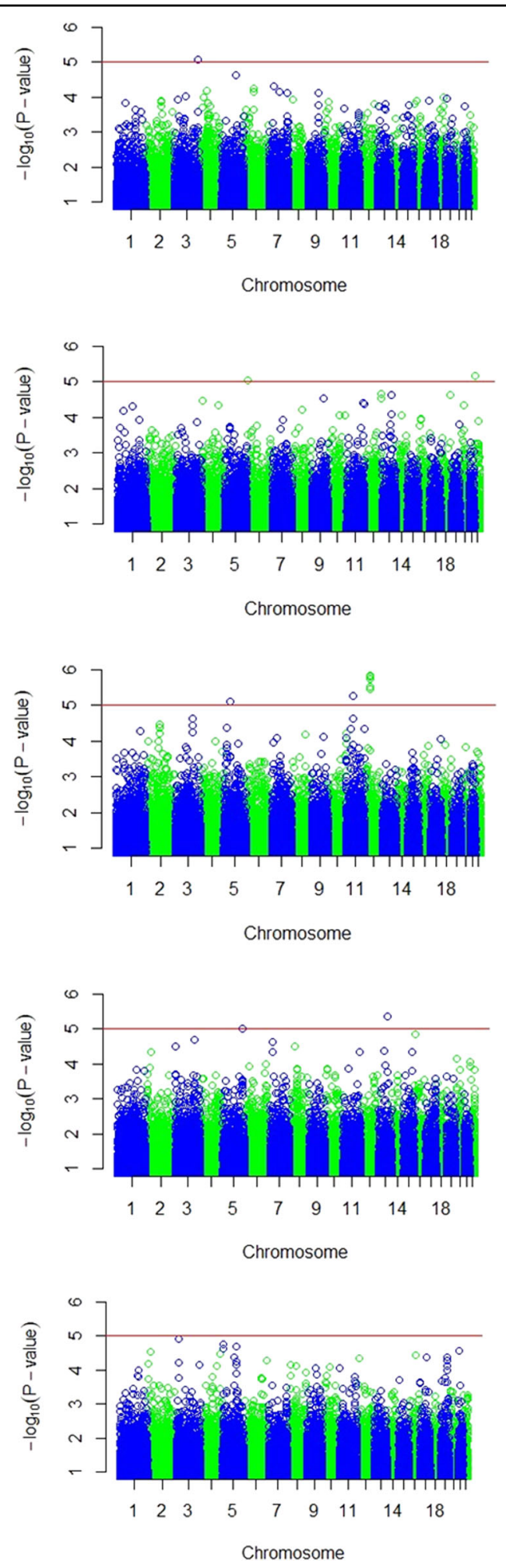

Fig. 1 Manhattan plots for the genome-wide association analyses of genetic variations and OCD dimensions. a Aggressive dimension. b Contamination dimension. c Order dimension. d Hoarding dimension. e Sexual/religious dimension. A blue line indicates the level of suggestive evidence of association $\left(p<1 \times 10^{-5}\right)$.

\section{Functional annotation}

Functional annotation was gathered for a final set of 154 (aggressive), 103 (contamination), 111 (order), 196 (hoarding) and 167 (sexual/religious) genes. The threshold used to select the genes included in the enrichment analyses $(p<0.01)$ included $1 \%$ of the genes in all the dimensions except the hoarding dimension, for which $2 \%$ of the genes presented a $p$-value lower than 0.01 .

Detailed information on the results of these analyses are given in Tables 4 and 5 . In the case of the hoarding dimension, the different pathways obtained are clustered in two groups according to their biological similarity.

\section{Discussion}

In this study, we examined the genomic bases of each of the most consistently validated symptom dimensions of OCD. Differential findings were obtained for the five dimensions considered. At the SNP-level, no variant reached genome-wide significance. The top SNPs were six markers in the order dimension (forming a single association peak) and one variant in the hoarding dimension $\left(p<5 \times 10^{-6}\right)$. Gene analyses showed one gene associated with hoarding (SETD3, $\left.p=1.89 \times 10^{-8}\right)$ and another with the aggressive dimension (CPE, $p=4.42 \times 10^{-6}$ ) at a genome-wide level. Different pathways or biological processes were represented by the aggressive, order, sexual/ religious, and hoarding dimensions. For contamination, no pathway remained associated after FDR correction.

Six of the top variants at the SNP-level analysis conformed a genomic region involving IPO8 and CAPRIN2, which presented an association signal with the order dimension. Certain intergenic variants near CAPRIN2 presented association signals $\left(p<5 \times 10^{-5}\right)$ with different neuropsychological variables and personality traits (https://www.ncbi.nlm.nih.gov/projects/gap/cgi-bin/study. cgi?study_id=phs000342.v18.p11; https://www.ncbi.nlm. nih.gov/projects/gap/cgi-bin/study.cgi?

study_id=phs000338.v1.p1). Considering the LD findings for this region and the fact that the six variants represent a single peak of association, it may be interesting to consider this genomic region in further studies, since it could be a relevant for the order dimension.

Gene-based analyses reported one gene associated with hoarding at the genome-wide significance level (SETD3, $\left.p=1.89 \times 10^{-8}\right)$. That gene is expressed in brain regions associated with $\mathrm{OCD}^{50,51}$ such as caudate and cerebellum (The Human Protein Atlas, SETD3, gene available from https://www.proteinatlas.org/ENSG000001835 76-SETD3/tissue) and seems to mediate transcriptome changes in the hypothalamus of mice ${ }^{52}$. It has also been associated with apoptotic processes, which in turn have been observed to mediate the neuronal loss in certain brain regions of BD patients ${ }^{53}$. Although two SNPs located near SETD3 show suggestive associations with autoimmune and 


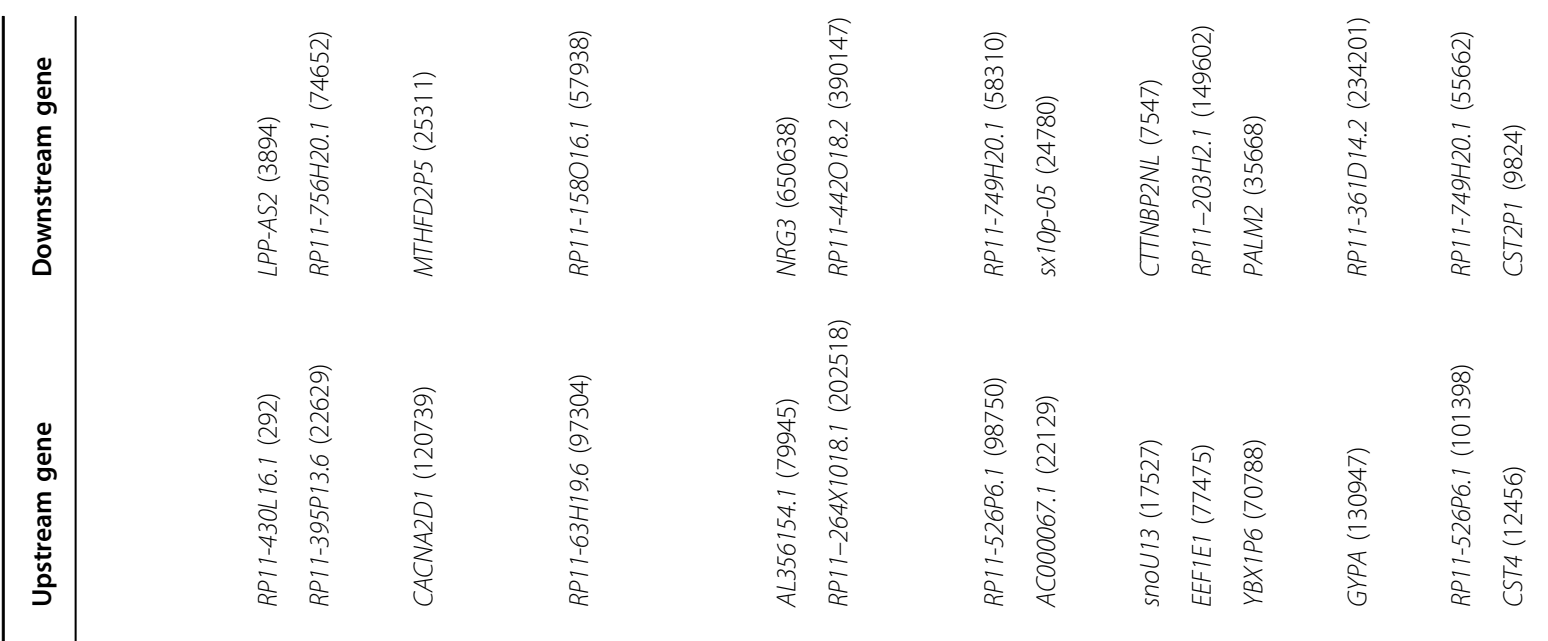

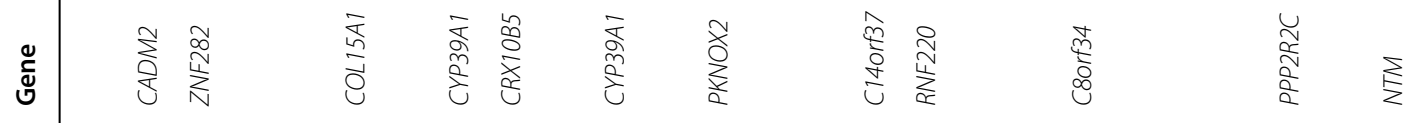

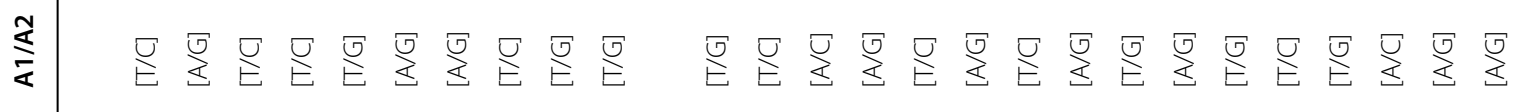

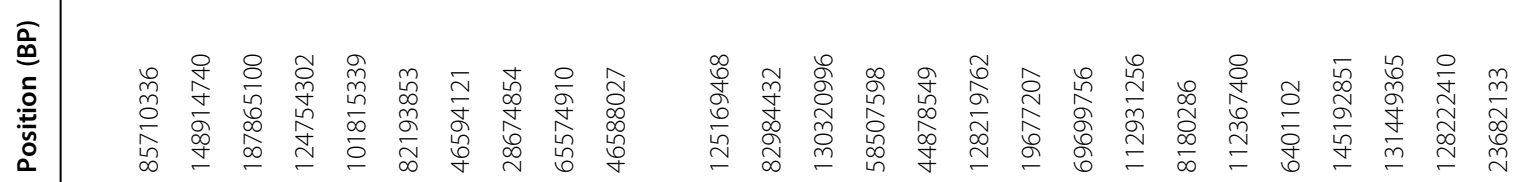

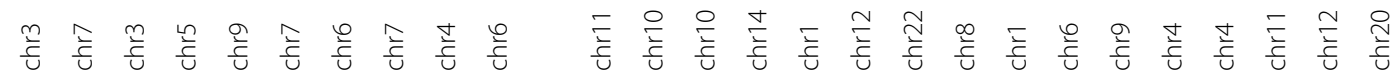

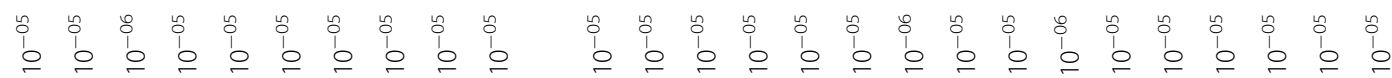

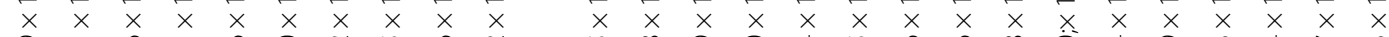

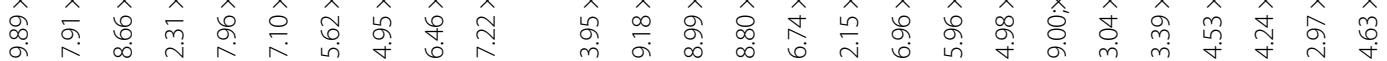

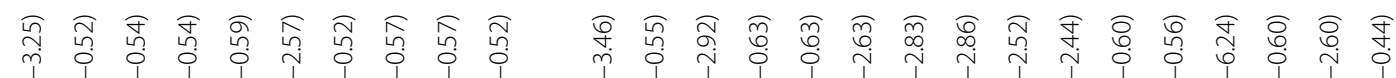

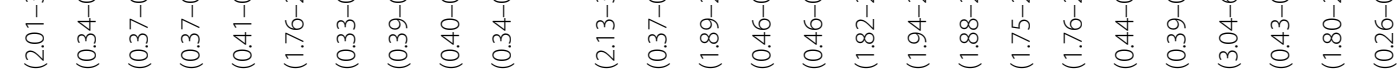
峲 f f

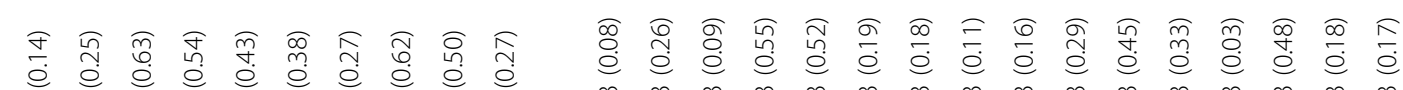

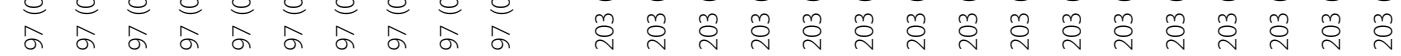

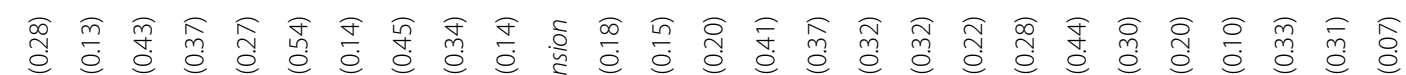

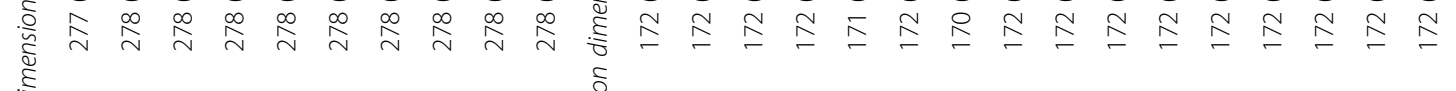

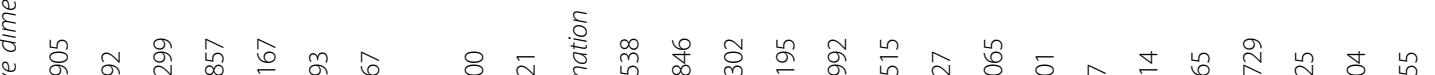

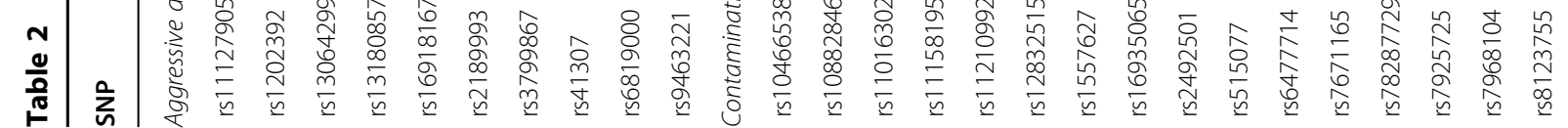




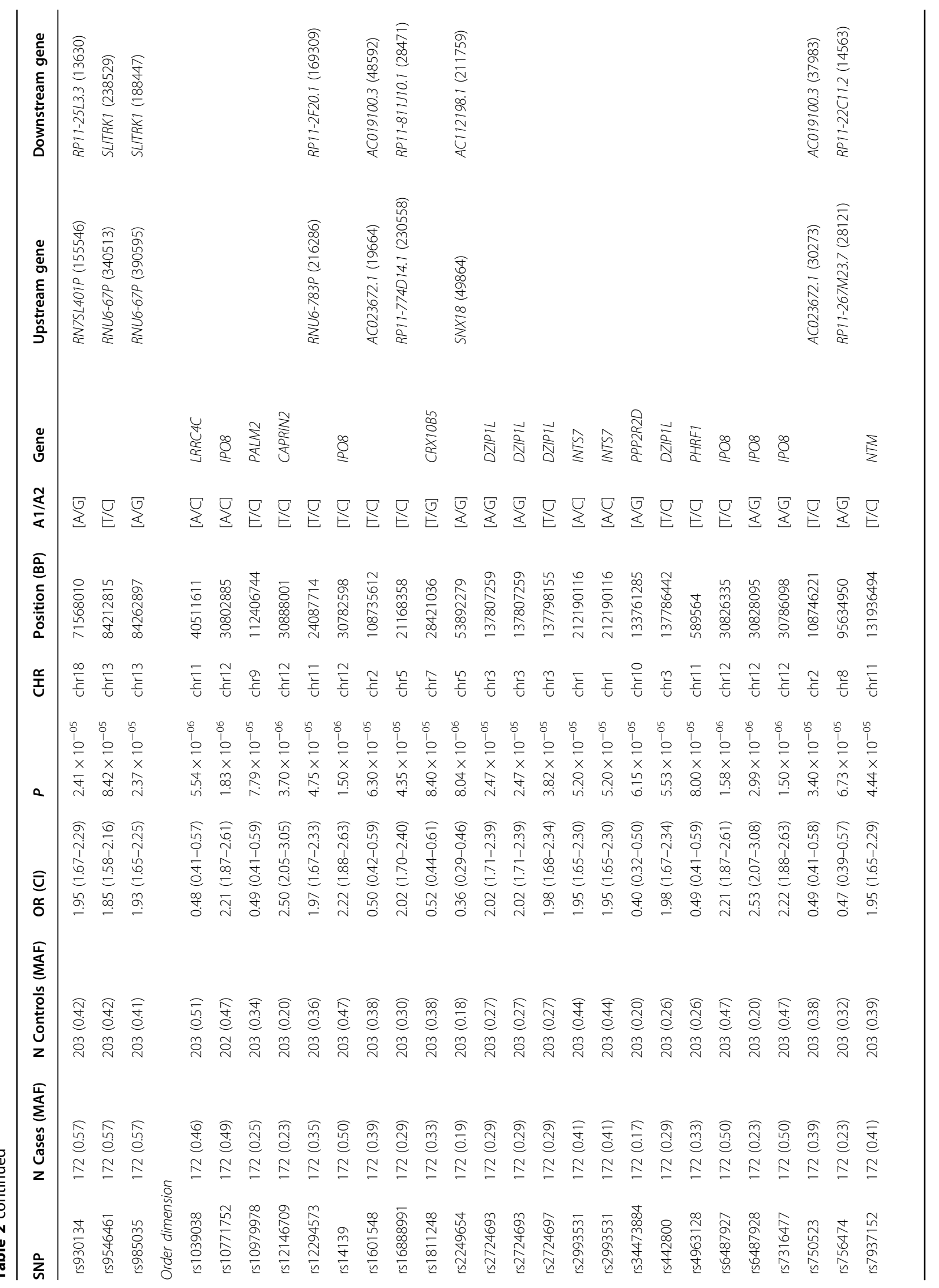




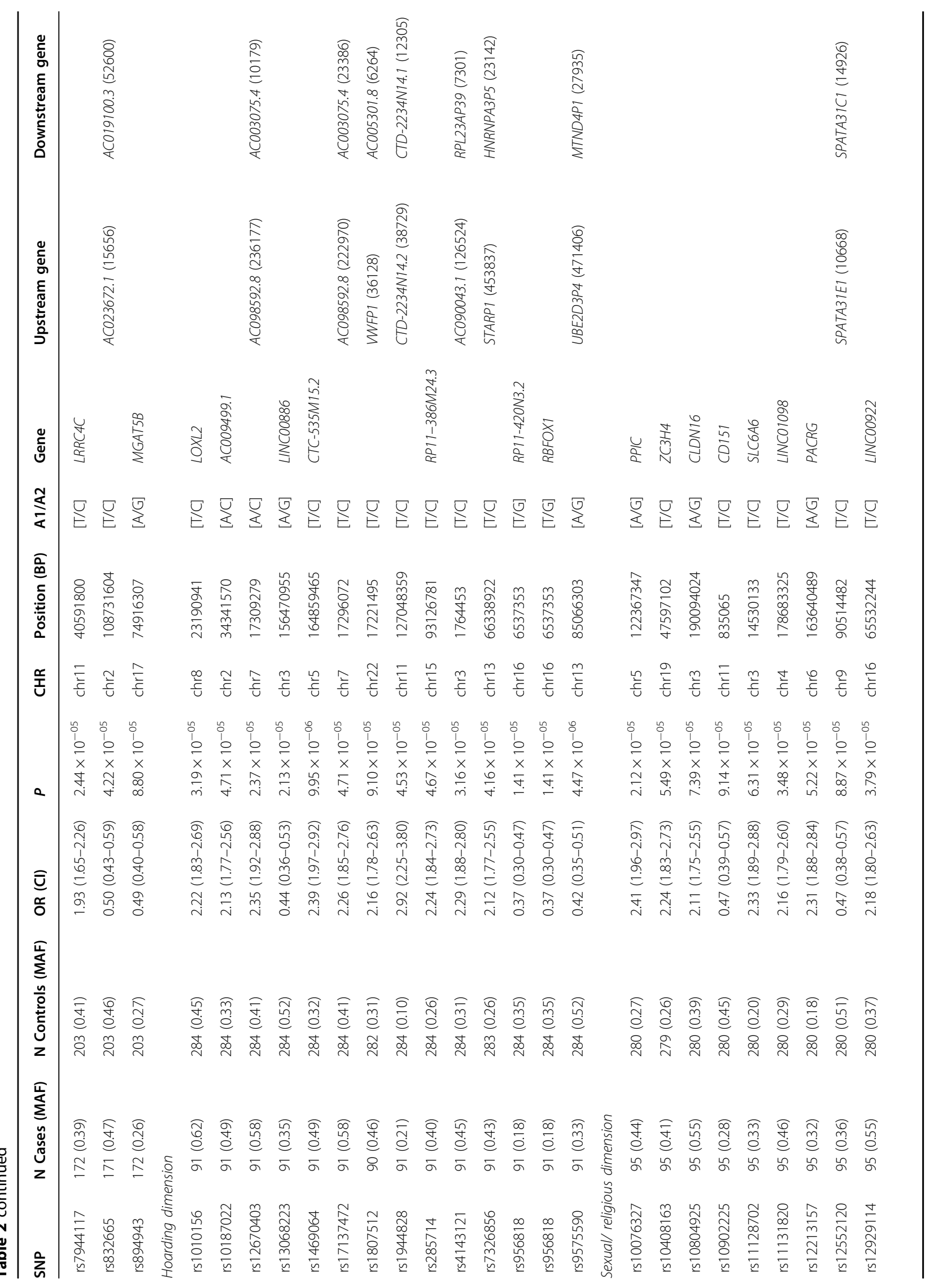




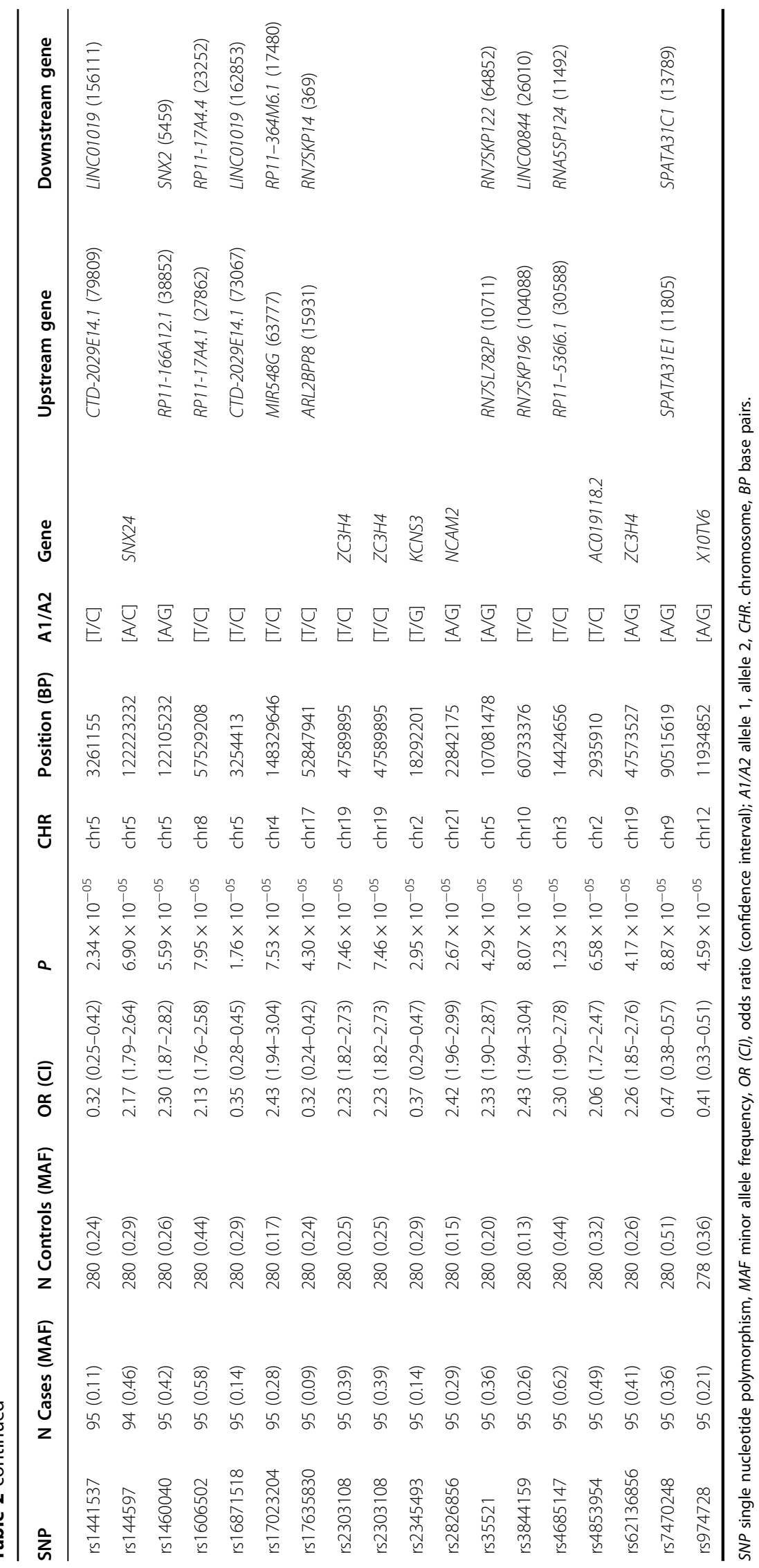




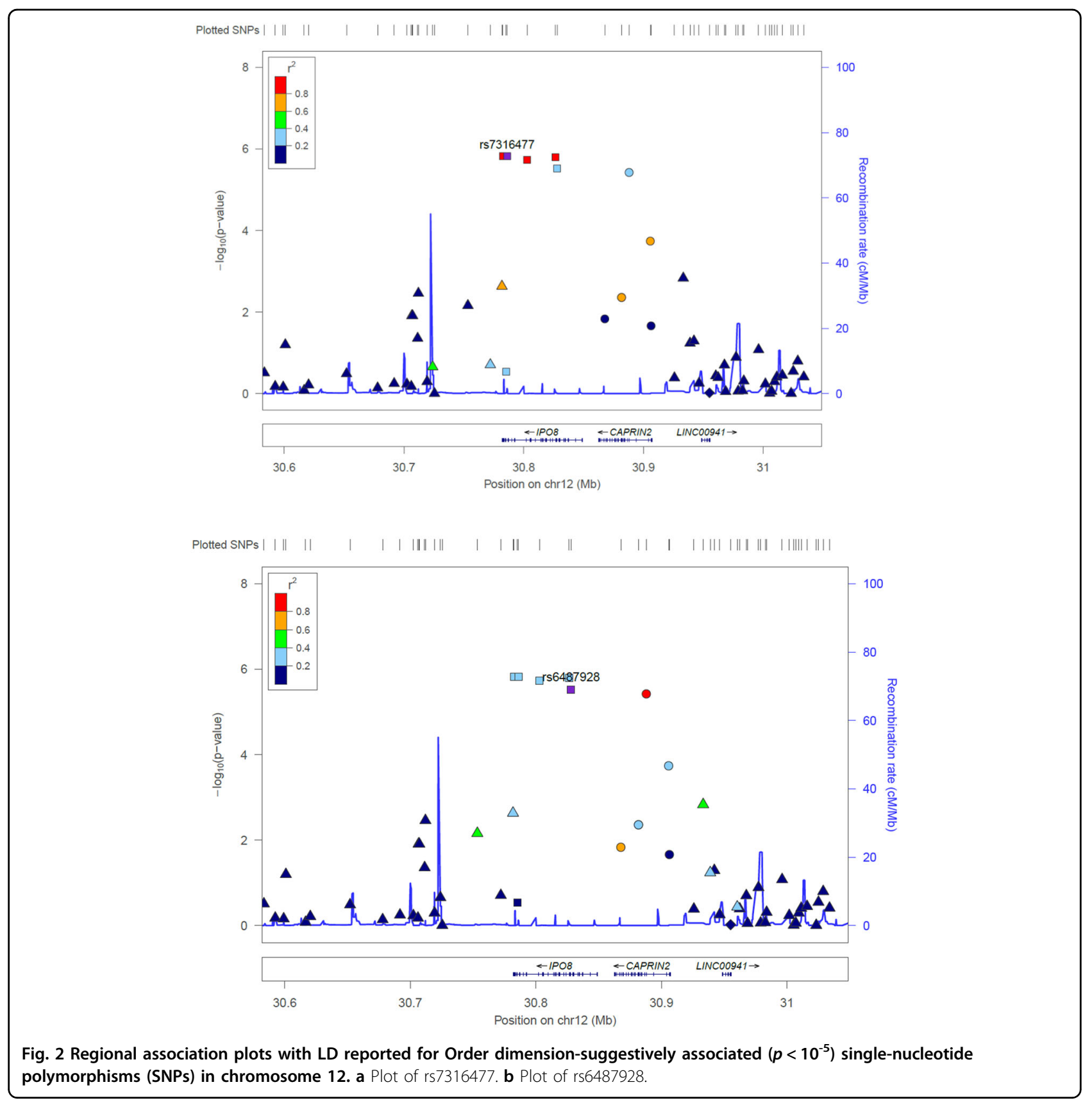

inflammatory conditions (rs2614463, $P=7.00 \times 10^{-6}$; rs2664299, $P=9.00 \times 10^{-6}$ ), none of them are in LD with any of the SETD3 variants in our analysis-rs12886549 $(P=0.68, \mathrm{MAF}=0.24, \mathrm{OR}=1.09) ;$ rs8015827 $(P=0.79$; $\mathrm{MAF}=0.39 ; \quad \mathrm{OR}=1.05) ; \quad \mathrm{rs} 34322735 \quad(P=1.72 \times 10-08$, $\mathrm{MAF}=0.01, \mathrm{OR}=101.39) . C P E$, which was associated with the aggressive dimension, is a gene highly expressed in brain $^{54}$. Different polymorphisms in this gene have been associated with a loss of neuroprotective function by reducing the effects of the oxidative stress in human cell lines $^{55}$ and transgenic mice ${ }^{56}$, leading to memory deficits and depressive behavior ${ }^{56}$. In addition, $C P E$-knockout mice have shown neurodegeneration in the hippocampus and the prefrontal cortex ${ }^{57}$.

In relation to the aggressive dimension, enrichment analyses showed overrepresentation in response to zinc ions (GO:0010043, FDR =0.002). Zinc ions are highly prevalent in the brain, being especially prominent in forebrain glutamatergic neurons, the hippocampus, and the amygdala ${ }^{58,59}$, and they plays an important role in neuronal plasticity ${ }^{60}$. Zinc deficiency has been associated with cognitive decline, Alzheimer's disease (AD) and 
Table 3 Best gene-based results from SKAT analyses for the different $O C D$ dimensions.

\begin{tabular}{llcll}
\hline Gene & $\boldsymbol{P}$ & QMETA & CMAF & NSNPS \\
\hline Aggressive & & & & \\
$\quad$ CPE & $\mathbf{4 . 4 2 \times 1 \mathbf { 1 0 } ^ { - 0 6 }}$ & 10857.8 & 4.18 & 15 \\
HIST1H2AH & $5.13 \times 10^{-05}$ & 9329.72 & 0.03 & 2 \\
FOXP4 & $1.01 \times 10^{-05}$ & 11790.03 & 1.48 & 8 \\
CREB5 & $9.29 \times 10^{-05}$ & 6440.17 & 2.87 & 12 \\
Order & & & & \\
PPP2R2D & $6.54 \times 10^{-05}$ & 23.49 & 0.65 & 3 \\
Hoarding & & & & \\
SERINC2 & $8.17 \times 10^{-05}$ & 10941.25 & 0.26 & 9 \\
SETD3 & $\mathbf{1 . 8 9} \times \mathbf{1 0}^{-\mathbf{0 8}}$ & 16579.98 & 0.64 & 3 \\
Sexual/Religious & & & & \\
CDC42BPA & $4.97 \times 10^{-05}$ & 7002.15 & 6.61 & 27 \\
GPR137B & $3.65 \times 10^{-05}$ & 18669.76 & 2.24 & 15 \\
LYZL4 & $3.48 \times 10^{-05}$ & 9965.91 & 0.07 & 3 \\
ABR & $9.13 \times 10^{-05}$ & 7283.78 & 1.81 & 7 \\
\hline
\end{tabular}

CMAF collected minor allele fruequency, QMETA test score reported by SKAT. Bold text highlights significant associations at a gene-based analysis level.

different psychiatric disorders in the elderly. In addition, genetic variants within ZNF142, a gene coding for a zincfinger protein, have been associated with a neurodevelopmental disorder resulting in speech impairment and intellectual disability ${ }^{61}$. The Peroxisomal lipid metabolism pathway (R-HSA-390918) was also significantly enriched in the aggressive dimension. Lipid metabolism has been extensively associated with different neuropsychiatric disorders, such as BD and depressive disorders, as well as $\mathrm{AD}^{62-65}$. Similarly, the sphingolipid signaling pathway (hsa04071) appears overrepresented in the order dimension. Sphingolipids are structural elements of cellular membranes and they play a role in cell signaling, differentiation and proliferation, apoptotic processes and inflammation ${ }^{66}$. Sphingolipid signaling has been observed to be involved in anxiety-like behavior in animal models as well as schizophrenia, depression and $\mathrm{BD}^{66-69}$.

Sexual/religious dimension genes were found enriched for $G$ alpha (12/13) signaling events (R-HSA-416482). G12/13 subunits are alpha units of heterotrimeric G proteins that regulate different cell processes through the use of guanine nucleotide exchange factors (GEFs). This family of G-protein subunits has been associated with neurodevelopment and is involved in processes of cell proliferation and migration ${ }^{70}$. In addition, G12 subunits have been observed to influence memory consolidation and contextual retrieval in mice, via increased expression in the hippocampus ${ }^{71}$. 


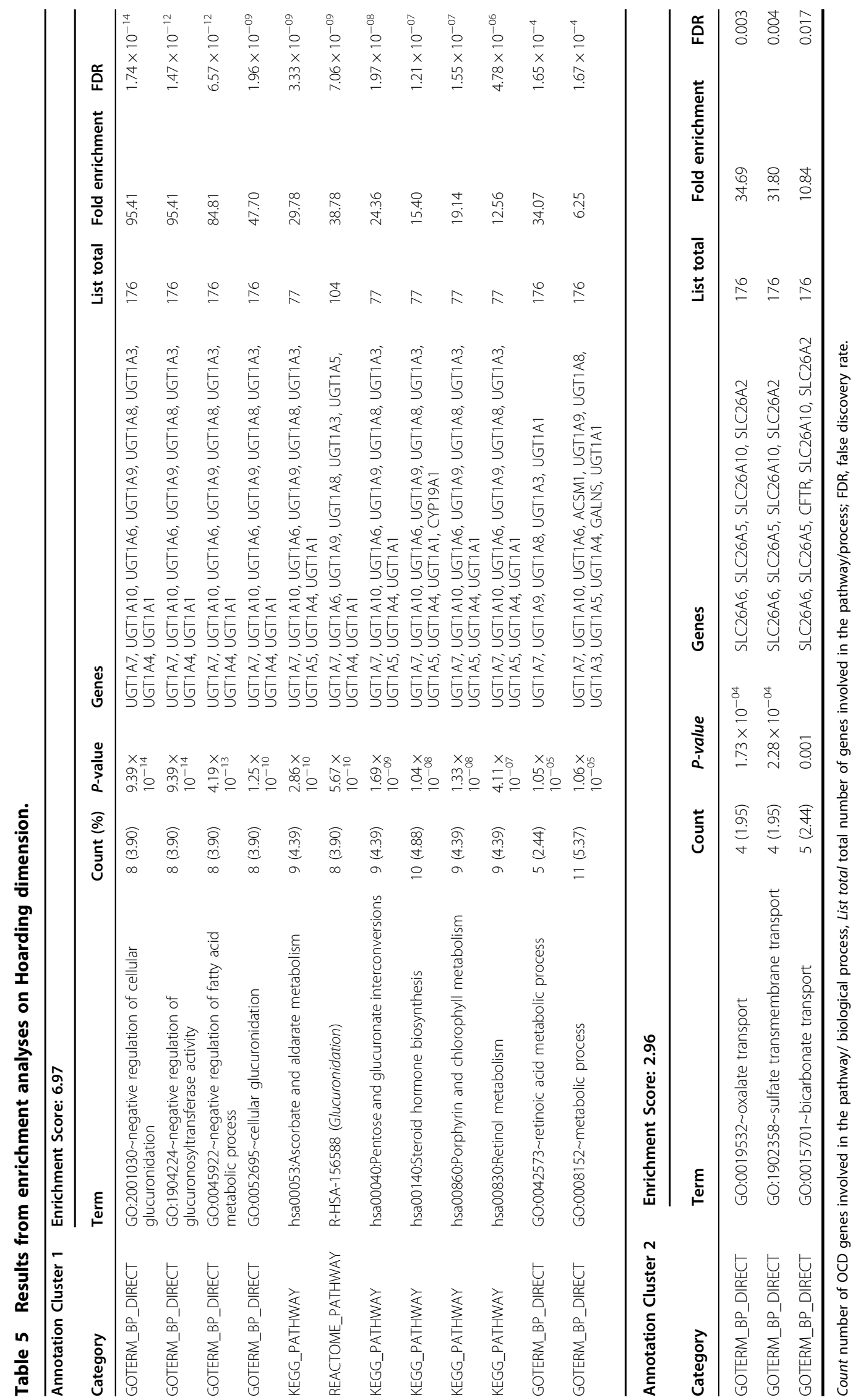


For the hoarding dimension, the first cluster of biological mechanisms and pathways includes cellular metabolic processes, such as lipid, vitamin and carbohydrate metabolism, all involving glucuronidation processes, as most genes included in these mechanisms or pathways code for UDP-glucuronosyltransferases (Table 5). It has been demonstrated that an alteration of the activity of these enzymes can affect brain function ${ }^{72}$. As an example, induction of UDP-glucuronosyltransferase $1 \mathrm{~A} 1$ during the prenatal period can cause neurodevelopmental disorders in mice ${ }^{73}$.

There is increasing evidence from metabolomic studies of the importance of metabolic processes in psychiatric disorders. Post-traumatic stress disorder (PTSD) has been associated with the alteration of different kinds of metabolites, such as monosaccharides, nucleosides or fatty $\operatorname{acids}^{74}$. Furthermore, there is evidence of dysfunctional metabolism of lipids and vitamins in depressed patients ${ }^{75}$, which may explain the high prevalence of comorbid metabolic syndrome and cardiovascular disease ${ }^{76}$.

A lower plasma concentration of certain lipids with a neuroprotective role also has been observed in PTSD patients, compared to healthy controls ${ }^{77}$. Altered lipid metabolism has been found in other psychiatric conditions, such as MDD, BD or schizophrenia, and associated with symptoms including anxiety, stress and cognitive impairment ${ }^{78}$. Lipid metabolism in turn influences steroid synthesis, which has been associated with brain electrical activity through the role of lipids in modulating neuronal excitability ${ }^{79}$. In addition, an alteration of porphyrin and chlorophyll metabolism might affect the formation of heme groups, possibly leading to neurotoxic effects, among others ${ }^{80,81}$.

The second cluster of biological mechanisms and pathways for the hoarding dimension is related to anion transport. Most of the hoarding dimension genes involved are members of the solute-carrier 26 family A (SLC26A). These transporters have been observed to influence, among other functions, microbiome composition, $\mathrm{pH}$ regulation, and anion transport ${ }^{82}$, which in turn have been related with the pathophysiology of different psychiatric disorders $^{83}$. More specifically, anion transport and $\mathrm{pH}$ regulation in the brain play a role in intra- and intersignaling and plasticity processes ${ }^{84}$. In relation to microbiome composition, late-onset autism has been associated with differences in the gastrointestinal microflora when compared to healthy controls ${ }^{85}$. Moreover, exposure to certain microbial pathogens during fetal development has been associated with the pathogenesis of schizophrenia in humans ${ }^{86}$, and both anxiety-like behavior and cognitive impairment in rodents ${ }^{87,88}$. It is interesting to note the increase in the genetic weight observed in the hoarding dimension when the rare variants are included in the analysis, since only two variants at the SNP level reach suggestive association and no SNP reaches genome-wide significance. This contrasts with the findings obtained for this dimension in the gene-based and pathway analyses, which are notably more numerous than for the other dimensions. We think this suggests a role for rare variants in the hoarding dimension. We also believe that the consistently observed higher heritability of this dimension, compared to the others ${ }^{9,10,89,90}$, could mostly be explained by the influence of rare variants. Further research is needed to reveal the genetic bases of the hoarding dimension.

Although OCD symptom dimensions overcome the unitary clinical diagnosis of $O C D$, subtyping $O C D$ according to overt clinical manifestations also presents significant limitations. Due to methodological differences, no concrete OCD dimensions classification system has been universally accepted. Some authors argue that other taxometric methods should be used to elucidate the symptom dimensions in OCD, including age of onset, comorbidities, or neuropsychological functioning in combination with clinical manifestations. The lack of significant associations among OCD symptom dimensions and individual SNPs could reflect limited statistical power due to the small sample size. Considering our total sample size, the number of cases and controls for each OCD dimension, a significance threshold of $p<2 \times 10^{-7}$ (Bonferroni threshold for 258,000 SNPs analyzed) and a risk allele frequency (MAF) of 0.1, our study has $80 \%$ power to detect a relative risk (RR) of 3.5 and 5 (equivalent to $\mathrm{OR}=4.8$ and $\mathrm{OR}=9$ ) for order and sexual/religious dimensions, respectively; for the three other $\mathrm{OCD}$ dimensions, the detectable RR under these conditions is 4 (equivalent to $\mathrm{OR}=6$ ). A representation of the statistical power achieved for different MAF and RR thresholds is shown in Supplementary Fig. 1 (Fig. S1a-e). Furthermore, we would have liked to consider the severity score for each dimension in our analysis, in addition to their presence/absence, which would have been possible with a larger sample. However, our sample was thoroughly characterized phenotypically, and our results highlight important differences in relation to the genetic bases, as well as the genetic load of the different OCD dimensions. In addition, rare variants are considered at gene-based and pathway analyses, since this kind of analysis increases the power of detecting small effects. The inclusion of rare variants is important given the growing appreciation for their importance in neuropsychiatric disorders ${ }^{91-93}$.

OCD is a highly heterogeneous disorder in terms of symptom profile, comorbidity and underlying brain substrate, which represents a challenge for understanding and treating the disorder. This heterogeneity may confound and contribute to mostly negative findings in current genome-wide analysis studies, despite clear evidence for a strong genetic component of the disorder based on twin 
and family studies, ranging from $40-65 \%$. Therefore, broad consensus has emerged for the need to explore OCD not as a homogeneous diagnosis, but rather considering other phenomenological approaches that investigate more refined phenotypes. In this sense, investigating genetic markers associated with different OCD symptom dimensions could be a useful strategy to begin disentangling the complex genetic vulnerability of the disorder. A clearer identification of susceptibility genes for OCD would translate into a better understanding of the etiology of the disorder and would help to develop potentially targeted and specific treatment approaches to improve the long-term outcome for OCD patients.

\section{Acknowledgements}

This study was supported in part by the Carlos III Health Institute (PI16/00950, PI18/00856); FEDER funds ('A way to build Europe') and by the Agency of University and Research Funding Management of the Catalan Government (2014SGR1672). M.A. was supported by the Secretariat for Universities and Research of the Ministry of Business and Knowledge of the Government of Catalonia. Grant co-funded by the European Social Fund (ESF) "ESF, Investing in your future" (2017 FI_B 00327). None of these funding sources participated in the preparation of the research article. We thank all the study participants from the Department of Psychiatry of Hospital Universitari de Bellvitge who collaborated to obtain the sample of this study.

\section{Author details}

'Institut d'Investigació Biomèdica de Bellvitge (IDIBELL), L'Hospitalet de Llobregat, Barcelona, Spain. ${ }^{2} \mathrm{OCD}$ Clinical and Research Unit, Psychiatry Department, Hospital Universitari de Bellvitge, Barcelona, Spain. ${ }^{3}$ Department of Clinical Sciences, Bellvitge Campus, University of Barcelona, Barcelona, Spain. ${ }^{4}$ Grupo de Medicina Xenómica, CIBERER, Centre for Research in Molecular Medicine and Chronic Diseases (CIMUS), Universidade de Santiago de Compostela, Santiago de Compostela, Spain. ${ }^{5}$ CIBERSAM (Centro de Investigación en Red de Salud Mental), Instituto de Salud Carlos III, Madrid, Spain. ${ }^{6}$ Institut de Recerca Sant Joan de Déu, Esplugues de Llobregat, Spain. ${ }^{7}$ Institut de Biomedicina de la Universitat de Barcelona (IBUB), CIBERER, and Dept. Genetics, Microbiology \& Statistics, Faculty of Biology, University of Barcelona, Barcelona, Spain. ${ }^{8}$ Fundación Pública Galega de Medicina Xenómica, SERGAS, Instituto de Investigación Sanitaria de Santiago de Compostela (IDIS), Santiago de Compostela, Spain

\section{Conflict of interest}

The authors declare that they have no conflict of interest.

\section{Publisher's note}

Springer Nature remains neutral with regard to jurisdictional claims in published maps and institutional affiliations.

Supplementary Information accompanies this paper at (https://doi.org/ 10.1038/s41398-020-0804-z).

Received: 29 October 2019 Revised: 23 March 2020 Accepted: 8 April 2020 Published online: 18 May 2020

\footnotetext{
References

1. Ruscio, A. M., Stein, D. J., Chiu, W. T. \& Kessler, R. C. The epidemiology of obsessive-compulsive disorder in the National Comorbidity Survey Replication. Mol. Psychiatry 15, 53-63 (2010).

2. Katerberg, H. et al. Symptom dimensions in OCD: Item-level factor analysis and heritability estimates. Behav. Genet 40, 505-517 (2010).
}

3. Schulze, D., Kathmann, N. \& Reuter, B. Getting it just right: a reevaluation of OCD symptom dimensions integrating traditional and Bayesian approaches. J. Anxiety Disord. 56, 63-73 (2018).

4. Bloch, M. H., Landeros-Weisenberger, A., Rosario, M. C., Pittenger, C. \& Leckman, J. F. Meta-analysis of the symptom structure of obsessive-compulsive disorder. Am. J. Psychiatry 165, 1532-1542 (2008).

5. Mataix-Cols, D., do Rosario-Campos, M. C. \& Leckman, J. F. A multidimensional model of obsessive-compulsive disorder. Am. J. Psychiatry 162, 228-238 (2005).

6. Rosario-Campos, M. C. et al. The Dimensional Yale-Brown Obsessive-Compulsive Scale (DY-BOCS): An instrument for assessing obsessive-compulsive symptom dimensions. Mol. Psychiatry 11, 495-504 (2006).

7. Brakoulias, V. et al. The familiality of specific symptoms of obsessivecompulsive disorder. Psychiatry Res 239, 315-319 (2016).

8. Stein, D. et al. Escitalopram in obsessive-compulsive disorder: response of symptom dimensions to pharmacotherapy. CNS Spectr. 13, 492-498 (2008).

9. Hasler, G. et al. Familiality of factor analysis-derived YBOCS dimensions in OCDaffected sibling pairs from the OCD Collaborative Genetics Study. Biol. Psychiatry 61, 617-625 (2007).

10. Cullen, B. et al. Factor analysis of the Yale-Brown obsessive compulsive scale in a family study of obsessive-compulsive disorder. Depress. Anxiety 24, 130-138 (2007).

11. Gilbert, A. R. et al. Brain structure and symptom dimension relationships in obsessive-compulsive disorder: A voxel-based morphometry study. J. Affect Disord. 109, 117-126 (2008).

12. Reess, T. J. et al. Association between hippocampus volume and symptom profiles in obsessive-compulsive disorder. Neurolmage Clin. 17, 474-480 (2018).

13. Okada, K. et al. Biological heterogeneity of obsessive-compulsive disorder: a voxel-based morphometric study based on dimensional assessment. Psychiatry Clin. Neurosci. 69, 411-421 (2015).

14. Via, E. et al. Amygdala activation and symptom dimensions in obsessivecompulsive disorder. Br. J. Psychiatry 204, 61-68 (2014).

15. Harrison, B. J. et al. Brain corticostriatal systems and the major clinical symptom dimensions of obsessive-compulsive disorder. Biol. Psychiatry 73, 321-328 (2013).

16. Domschke, K. \& Dannlowski, U. Imaging genetics of anxiety disorders. Neuroimage 53, 822-831 (2010).

17. Alexopoulos, G. S. et al. Serotonin transporter polymorphisms, microstructural white matter abnormalities and remission of geriatric depression. J. Affect Disord. 13, 1519-1523 (2010).

18. Atmaca, M. et al. The association of myelin oligodendrocyte glycoprotein gene and white matter volume in obsessive-compulsive disorder. J. Affect Disord. 124, 309-313 (2010).

19. Wolf, C. et al. CACNA1C genotype explains interindividual differences in amygdala volume among patients with schizophrenia. Eur. Arch. Psychiatry Clin. Neurosci. 264, 93-102 (2014).

20. Tesli, M. et al. CACNA1C Risk variant and amygdala activity in bipolar disorder, schizophrenia and healthy controls. PLOS ONE 8, 6-11. (2013).

21. $M J$, T. R. J. et al. BDNF gene and obsessive compulsive disorder risk, symptom dimensions and treatment response. Asian J. Psychiatr. 38, 65-69 (2018).

22. Lennertz, L. et al. 5-HT3 receptor influences the washing phenotype and visual organization in obsessive-compulsive disorder supporting 5-HT3 receptor antagonists as novel treatment option. Eur. Neuropsychopharmacol. 24, 86-94 (2014).

23. Melo-Felippe, F. B., Fontenelle, L. F. \& Kohlrausch, F. B. Gene variations in PBX1, LMX1A and SLITRK1 are associated with obsessive-compulsive disorder and its clinical features. J. Clin. Neurosci. 61, 180-185 (2019).

24. Melo-Felippe, F. B. et al. Catechol-O-methyltransferase gene polymorphisms in specific obsessive-compulsive disorder patients' subgroups. J. Mol. Neurosci. 58, 129-136 (2016).

25. Alonso, P. et al. Variants in estrogen receptor alpha gene are associated with phenotypical expression of obsessive-compulsive disorder. Psychoneuroendocrinology 36, 473-483 (2011).

26. Hasler, G., Kazuba, D. \& Murphy, D. L. Factor analysis of obsessive-compulsive disorder YBOCS-SC symptoms and association with 5-HTTLPR SERT polymorphism. Am. J. Med Genet Part B Neuropsychiatr. Genet 141, $403-408$ (2006).

27. Reshma, J. et al. DRD4 gene and obsessive compulsive disorder: do symptom dimensions have specific genetic correlates? Prog. Neuro-Psychopharmacol. Biol. Psychiatry 41, 18-23 (2013). 
28. Kohlrausch, F. B. et al. Association of GRIN2B gene polymorphism and obsessive compulsive disorder and symptom dimensions: a pilot study. Psychiatry Res 243, 152-155 (2016).

29. Kim, S. J., Lee, H. S. \& Kim, C. H. Obsessive-compulsive disorder, factor-analyzed symptom dimensions and serotonin transporter polymorphism. Neuropsychobiology 52, 176-182 (2005).

30. Cavallini, M. C., Di Bella, D., Siliprandi, F., Malchiodi, F. \& Bellodi, L. Exploratory factor analysis of obsessive-compulsive patients and association with 5HTTLPR polymorphism. Am. J. Med Genet 114, 347-353 (2002).

31. Alonso, P. et al. Genetic susceptibility to obsessive-compulsive hoarding: the contribution of neurotrophic tyrosine kinase receptor type 3 gene. In: mGenes, Brain Behav. 7, 778-785 (2008).

32. Sinopoli, V. M. et al. Serotonin system genes and obsessive-compulsive trait dimensions in a population-based, pediatric sample: a genetic association study. J. Child Psychol. Psychiatry Allied Discip. 12, 1289-1299 (2019).

33. Katerberg, $\mathrm{H}$. et al. The role of the brain-derived neurotrophic factor (BDNF) val66met variant in the phenotypic expression of Obsessive-Compulsive Disorder (OCD). Am. J. Med Genet Part B Neuropsychiatr. Genet 150, 1050-1062 (2009).

34. Bloch, M. H. \& Pittenger, C. The genetics of obsessive-compulsive disorder. Curr. Psychiatry Rev. 6, 91-103 (2010).

35. Lecrubier, Y. Refinement of diagnosis and disease classification in psychiatry. Eur. Arch. Psychiatry Clin. Neurosci. 258, 6-11 (2008).

36. Waszczuk, M. A., Kotov, R., Ruggero, C., Gamez, W. \& Watson D. Supplemental material for hierarchical structure of emotional disorders: from individual symptoms to the spectrum. J. Abnorm. Psychol. https://doi.org/10.1037/ abn0000264.supp (2017).

37. American Psychiatric Association. DSM-N-TR: Diagnostic and Statistical Manual of Mental Disorders, 4th Ed. (American Psychiatric Press: Washington, DC, 1994).

38. First, M. B., Spitzer, R. L., Gibbon, M. \& Williams J. B. W. Structured Clinical Interview for DSM-IV Axis I Disorders-Clinician Version (SCID-CV) (1996).

39. Goodman, W. K. et al. The Yale-Brown Obsessive Compulsive Scale. I. Development, use, and reliability. Arch. Gen. Psychiatry 46, 1006-1011 (1989).

40. Purcell, S. et al. PLINK: a tool set for whole-genome association and population-based linkage analyses. Am. J. Hum. Genet 81, 559-575 (2007).

41. Marees A. T. et al. A tutorial on conducting genome-wide association studies: quality control and statistical analysis. Int. J. Methods Psychiatr. Res. https://doi. org/10.1002/mpr.1608 (2018)

42. Aulchenko, Y. S., Ripke, S., Isaacs, A. \& van Duijn, C. M. GenABEL: An R library for genome-wide association analysis. Bioinformatics https://doi.org/10.1093/ bioinformatics/btm108 (2007).

43. Pruim, R. et al. LocusZoom: regional visualization of genome-wide association scan results. Bioinformatics https://doi.org/10.1093/bioinformatics/btq419 (2010).

44. Wu, M. C. et al. Rare-variant association testing for sequencing data with the sequence kernel association test. Am. J. Hum. Genet 89, 82-93 (2011).

45. Voorman, A., Brody, J. \& Lumley T. SkatMeta: an R package for meta analyzing region-based tests of rare DNA variants (2013).

46. Huang, D. W., Sherman, B. T. \& Lempicki, R. A. Systematic and integrative analysis of large gene lists using DAVID Bioinformatics Resources. Nat. Protoc. 4, 44-57 (2009).

47. Huang, D. W., Sherman, B. T. \& Lempicki, R. A. Bioinformatics enrichment tools: paths toward the comprehensive functional analysis of large gene lists. Nucleic Acids Res 37, 1-13 (2009).

48. Kwiatkowski, S. et al. SETD3 protein is the actin-specific histidine Nmethyltransferase. https://doi.org/10.7554/eLife.37921.001 (2018).

49. Xiao, L., Yang, X. \& Loh, Y. P. Neurotrophic, gene regulation, and cognitive functions of carboxypeptidase E-neurotrophic factor-a1 and its variants. Front Neurosci https://doi.org/10.3389/fnins.2019.00243 (2019).

50. Burguière, E., Monteiro, P., Mallet, L., Feng, G. \& Graybiel, A. M. Striatal circuits, habits, and implications for obsessive-compulsive disorder. Curr. Opin. Neurobiol. 30, 59-65 (2015).

51. Hazari, N., Narayanaswamy, J. \& Venkatasubramanian, G. Neuroimaging findings in obsessive-compulsive disorder: a narrative review to elucidate neurobiological underpinnings. Indian J. Psychiatry 61, S9-S29 (2019).

52. De Giorgio, M. R., Yoshioka, M. \& St-Amand, J. Feeding induced changes in the hypothalamic transcriptome. Clin. Chim. Acta 406, 103-107 (2009).

53. Scaini, G. et al. Perturbations in the apoptotic pathway and mitochondrial network dynamics in peripheral blood mononuclear cells from bipolar disorder patients. Transl. Psychiatry 7, e1111 (2017).
54. Fagerberg, L. et al. Analysis of the human tissue-specific expression by genome-wide integration of transcriptomics and antibody-based proteomics. Mol. Cell Proteom. 13, 397-406 (2014).

55. Cong, L., Cheng, Y., Cawley, N. X., Murthy, S. R. K. \& Loh, Y. P. A novel single nucleotide $\mathrm{T} 980 \mathrm{C}$ polymorphism in the human carboxypeptidase $\mathrm{E}$ gene results in loss of neuroprotective function. PLoS One 12, 1-12. (2017).

56. Cheng, Y. et al. A human carboxypeptidase E/NF-al gene mutation in an Alzheimer's disease patient leads to dementia and depression in mice. Transl. Psychiatry 6, e973-12 (2016).

57. Cheng, Y., Cawley, N. X. \& Loh, Y. P. Carboxypeptidase E (NF-a1): a new trophic factor in neuroprotection. Neurosci. Bull. 30, 692-696 (2014).

58. Grønli O., Kvamme J. M., Friborg O. \& Wynn R. Zinc deficiency is common in several psychiatric disorders. PLOS ONE https:/doi.org/10.1371/journal. pone.0082793 (2013)

59. Portbury, S. D. \& Adlard P. A. Zinc signal in brain diseases. Int. J. Mol. Sci. https:// doi.org/10.3390/ijms18122506 (2017)

60. McAllister, B. B. \& Dyck, R. H. Zinc transporter 3 (ZnT3) and vesicular zinc in central nervous system function. Neurosci. Biobehav. Rev. 80, 329-350 (2017).

61. Khan, K. et al. Recessive variants in ZNF142 cause a complex neurodevelopmental disorder with intellectual disability, speech impairment, seizures, and dystonia. Genet. Med. https://doi.org/10.1038/s41436-019-0523-0 (2019).

62. D'Ambrosio, V., Salvi, V., Bogetto, F. \& Maina, G. Serum lipids, metabolic syndrome and lifetime suicide attempts in patients with bipolar disorder. Prog Neuro-Psychopharmacol. Biol. Psychiatry 37, 136-140 (2012).

63. Heckbert, S. R. et al. Depression in relation to long-term control of glycemia, blood pressure, and lipids in patients with diabetes. J. Gen. Intern Med $\mathbf{2 5}$ 524-529 (2010)

64. Yadav, R. S. \& Tiwari, N. K. Lipid Integration in neurodegeneration: an overview of alzheimer's disease. Mol. Neurobiol. 50, 168-176 (2014).

65. Grela, A. et al. Application of fatty acid and lipid measurements in neuropsychiatry. Clin. Chem. Lab. Med. 54, 197-206. (2016).

66. Narayan, S. \& Thomas, E. Sphingolipid abnormalities in psychiatric disorders: a missing link in pathology? Front Biosci. 16, 1797 (2011).

67. Mühle, C., Reichel, M., Gulbins, E. \& Kornhuber J. in Sphingolipids in Disease (eds Gulbins Erich \& Petrache I.) 431-456 (Springer Vienna: Vienna, 2013).

68. Schwarz, E. et al. High throughput lipidomic profiling of schizophrenia and bipolar disorder brain tissue reveals alterations of free fatty acids, phosphatidylcholines, and ceramides. J. Proteome Res 7, 4266-4277 (2008).

69. Kornhuber, J. et al. High activity of acid sphingomyelinase in major depression. J. Neural Transm. 112, 1583-1590 (2005).

70. Iguchi, $T$. et al. Orphan G protein-coupled receptor GPR56 regulates neural progenitor cell migration via a Ga12/13 and Rho pathway. J. Biol. Chem. 283 14469-14478 (2008)

71. Zelek-Molik, A. et al. Fear memory-induced alterations in the mRNA expression of $G$ proteins in the mouse brain and the impact of immediate posttraining treatment with morphine. Prog. Neuro-Psychopharmacol. Biol. Psychiatry 93 221-231 (2019)

72. Grancharov, K., Naydenova, Z., Lozeva, S. \& Golovinsky, E. Natural and synthetic inhibitors of UDP-glucuronosyltransferase. Pharmacol. therapeut. 89, 171-186 (2001).

73. Hirashima, R. et al. Induction of the UDP-Glucuronosyltransferase 1A1 during the Perinatal Period Can Cause Neurodevelopmental Toxicity. Mol. Pharm. 90 265-274 (2016).

74. Karabatsiakis, A. et al. Metabolite profiling in posttraumatic stress disorder. J. Mol. Psychiatry 3, 2 (2015).

75. Maes, M. An intriguing and hitherto unexplained co-occurrence: Depression and chronic fatigue syndrome are manifestations of shared inflammatory, oxidative and nitrosative (IO\&NS) pathways. Prog. Neuro-Psychopharmacol. Biol. Psychiatry 35, 784-794 (2011).

76. de Melo, L. G. P. et al. Shared metabolic and immune-inflammatory, oxidative and nitrosative stress pathways in the metabolic syndrome and mood disorders. Prog. Neuro-Psychopharmacol. Biol. Psychiatry 78, 34-50 (2017).

77. Mellon, S. H., Gautam, A., Hammamieh, R., Jett, M. \& Wolkowitz, O. M. Metabolism, metabolomics, and inflammation in posttraumatic stress disorder. Biol. Psychiatry 83, 866-875 (2018).

78. Perica, M. M. \& Delaš, I. Essential fatty acids and psychiatric disorders. Nutr. Clin. Pract. 26, 409-425 (2011).

79. Joëls, M. Steroid hormones and excitability in the mammalian brain. Front Neuroendocrinol. 18, 2-48 (1997).

80. Gozzelino, R. The pathophysiology of heme in the brain. Curr. Alzheimer Res $\mathbf{1 3}$ 174-184 (2016). 
81. Bonkovsky, H. L. et al. Porphyrin and heme metabolism and the porphyrias. Compr. Physiol. 3, 365-401. (2013)

82. Seidler, U. \& Nikolovska, K. Slc26 Family of anion transporters in the gastrointestinal tract: expression, function, regulation, and role in disease. Compr. Physiol. 9, 839-872 (2019).

83. Malan-Muller, S. et al. The gut microbiome and mental health: implications for anxiety- and trauma-related disorders. Omi A J. Integr. Biol. 22, 90-107 (2018).

84. Bonnet, U., Bingmann, D., Speckmann, E. J. \& Wiemann, M. Levetiracetam mediates subtle pH-shifts in adult human neocortical pyramidal cells via an inhibition of the bicarbonate-driven neuronal pH-regulation - Implications for excitability and plasticity modulation. Brain Res 1710, 146-156 (2019).

85. Finegold, S. M. et al. Gastrointestinal Microflora Studies in Late-Onset Autism. Clin. Infect. Dis. 35, S6-S16. (2002).

86. Mittal, V. A., Ellman, L. M. \& Cannon, T. D. Gene-environment interaction and covariation in schizophrenia: the role of obstetric complications. Schizophr. Bull. 34, 1083-1094 (2008).

87. Goehler, L. E., Park, S. M., Opitz, N., Lyte, M. \& Gaykema, R. P. A. Campylobacter jejuni infection increases anxiety-like behavior in the holeboard: possible anatomical substrates for viscerosensory modulation of exploratory behavior. Brain Behav. Immun. 22, 354-366 (2009).

88. Bilbo, S. D. et al. Neonatal infection induces memory impairments following an immune challenge in adulthood. Behav. Neurosci. 119, 293-301 (2005).

89. Iervolino A. C. et al. Article Prevalence and Heritability of Compulsive Hoarding: A Twin Study. http://www.vcu.edu/mx/ (2009)

90. Mathews, C. A. et al. Heritability and clinical features of multigenerational families with obsessive-compulsive disorder and hoarding. Am. J. Med Genet Part B Neuropsychiatr. Genet 144, 174-182 (2007).

91. Ament, S. A. et al. Rare variants in neuronal excitability genes influence risk for bipolar disorder. Proc. Natl Acad. Sci. USA 112, 3576-3581 (2015).

92. Amin $\mathrm{N}$. et al. A rare missense variant in RCL1 segregates with depression in extended families. Mol. Psychiatry https://doi.org/10.1038/mp.2017.49 (2018)

93. Singh T. et al. The contribution of rare variants to risk of schizophrenia in individuals with and without intellectual disability. Nat Genet. https:/doi.org/ 10.1038/ng.3903 (2017). 